\title{
Comparative study of psoas compartment block and sciatic nerve block with that of spinal block anesthesia for lower extremity surgeries
}

\author{
Prerana Jogdand ${ }^{1}$, Prasad M. Sule ${ }^{2, *}$ \\ Assistant Professor, Dept. of Anaesthesia, S.R.T.R. Government Medical College, Ambajogai, Maharashtra, India \\ *Corresonding Author: \\ Email: prasadsule.gmcl@gmail.com
}

Received: $29^{\text {th }}$ June, 2017

Accepted: $06^{\text {th }}$ September, 2017

\begin{abstract}
Aim and Objectives: Aim of the present study was to compare the efficacy of the block, hemodynamic effects, postoperative analgesia and side effects of combined psoas compartment sciatic nerve block with continuous spinal anaesthesia in adult patients undergoing lower extremity surgeries.

Materials and Methods: Sixty patients of either sex, ASA grade I, II and III, age between 18-70 years, were randomly allocated into two groups. In group A, psoas compartment block was performed by posterior approach using $30 \mathrm{cc}$ of $0.25 \%$ bupivacaine and sciatic nerve block by classical posterior approach using the $20 \mathrm{cc}$ of $0.25 \%$ bupivacaine. In group B, continuous spinal anaesthesia was performed in the L3-L4 interspaced with the patient in lateral decubitus position using $3 \mathrm{cc}(15 \mathrm{mg})$ of $0.5 \%$ heavy bupivacaine. We studied various parameters of block, hemodynamic effects, duration of postoperative analgesia and any complications.

Results: Group B had earlier onset of sensory and motor block as compare to group A. The mean duration of sensory and motor block was significantly prolonged in group A than in group B. The efficacy of sensory blockade was comparable in both the groups while in group A $90 \%$ patients and in group B $97 \%$ of patients had complete motor blockade. The incidence of hypotension was more with group B as compared to group A. The duration of post-operative analgesia was longer in group A than in group B. No major complications were observed in both the groups.

Conclusions: It was concluded that combined psoas compartment block and sciatic nerve block is a beneficial alternative to existing anaesthesia techniques for lower extremities surgeries.
\end{abstract}

Keywords: Bupivacaine, Combined psoas compartment-sciatic nerve block, Continuous spinal anaesthesia, Lower extremity surgeries, Post-operative analgesia, Sensory and motor blockade.

\section{Introduction}

The lower extremity surgeries involve fractures of hip, femur; tibia, ankle surgeries, lower limb amputation and these surgeries are very common in geriatric age group which comprises about $7 \%$ of total population. ${ }^{1}$ These patients associated with increased incidence of cardio-respiratory problems posted for surgical repair of hip fractures impose challenges to anaesthesiologist to maintain homeostasis with good surgical anaesthesia. Usually when such cases are confronted, the choice of anaesthesia would be general anaesthesia or regional specifically spinal anaesthesia, epidural anaesthesia and lumbar plexus blockade.

The regional anaesthesia in form of central neuroaxial blockade is considered to be a favored technique for lower limb fracture surgeries but it is associated with hemodynamic instability and cardiorespiratory problems in very old patients. ${ }^{2}$ There is a high-risk of failure in the implementation of these methods $^{3}$ because these methods are technically complicated.

During surgery continuous spinal anaesthesia (CSA) provides extension of blockage and versatile pain management during the postoperative period via an indwelling catheter allowed intermittent local anaesthetic injection into the subarachnoid space. ${ }^{4}$ The existing cardiac disease and intravascular volume status affect the extent of sympathetic blockade which may lead to adverse hemodynamic changes by CSA. Also, CSA was found to be associated with a high incidence of post dural puncture headache (PDPH). ${ }^{5}$ To conquer these side effect different anaesthetic technique like combined psoas compartment-sciatic nerve block (PCSNB) was used.

Lower limb is supplied by nerves of sacral plexus and lumbar plexus, so blocking these nerves also provides anaesthesia for lower limbs. Although Lumbar plexus block is a good option but it is rarely accepted because of technical difficulties. Considering pitfalls of central neuroaxial anaesthesia, it is hypothesised that lumbar plexus block with sciatic nerve block can be a promising regional technique that provides adequate anaesthesia with lesser rate of complications with stable hemodynamics.

Therefore, this prospective randomised study was designed to compare the quality of anesthesia and hemodynamic changes of PCSNB with CSA in adult patients undergoing extremity surgeries.

\section{Materials and Methods}

The prospective, randomized study was conducted after obtaining the approval of institutional ethical 
committee and written informed consent from all patients. 60 adult patients of either sex, age between 18 to 70 years, ASA grade I, II and III, scheduled for lower extremity surgeries were included for the study. Patients having own refusal for participation, patient with bleeding disorder, infection at the site of the block, sensitivity to the local anaesthetic, other contraindications of psoas compartment block include vertebral and meningeal infectious syndromes, lumbar vertebral trauma and significant scoliosis, ${ }^{6}$ also having relative contraindications include sepsis, psychiatric illness, anxious or agitated patient, diabetic neuropathy and neurologic lesion in the lumbar plexus region were excluded from the study. A comprehensive preanaesthetic evaluation was done for all the patients including case history, thorough clinical examination and all relevant investigations. Lignocaine sensitivity test was done prior to the procedure in which 1cc of plain lignocaine was injected intradermally, after 10 min the injection was watched for indurations or wheal. 60 adult patients were randomly allocated into two groups equally before transfer to the operating room. Group A (Psoas compartment block and sciatic nerve block): received psoas compartment block through femoral nerve block and sciatic nerve block with the help of peripheral nerve locator. Psoas compartment block was performed using $30 \mathrm{cc}$ of $0.25 \%$ bupivacaine while the sciatic nerve block was performed using $20 \mathrm{cc}$ of $0.25 \%$ bupivacaine. Maximum dosage was $2 \mathrm{mg} / \mathrm{kg}$ body weight. Group B received spinal anesthesia with 3 cc (15 mg) of $0.5 \%$ heavy bupivacaine.

All the patients were transported to the operation theater and baseline parameters were noted by means of standard monitors like ECG, pulse oximeter and non- invasive blood pressure. A good intravenous line was established with 18 or $20 \mathrm{G}$ cannula and started IV fluid. All patients received acid prophylaxis in form of injection ranitidine $50 \mathrm{mg}$ and metoclopramide $10 \mathrm{mg}$ intravenously. The patients in both groups were premedicated midazolam $0.03 \mathrm{mg} / \mathrm{kg}$ and pentazocine $0.3 \mathrm{mg} / \mathrm{kg}$ intravenously. Drugs necessary for resuscitation and anaesthesia administration and all the equipments were kept ready.

The posterior approach was performed for Psoas compartment block in group A. The patient was positioned in the lateral decubitus pose with the side to be operated uppermost and knees flexed. A line was drawn connecting both the iliac crests that crossed the lumbar body of the third lumbar nerve (L3). Another line was drawn from the posterosuperior iliac spine (PSIS) cephalad just parallel to the vertebral column. The juncture of the two lines denotes the most lateral section of the lumbar plexus. Under all safety measures, the insulated needle $(21 \mathrm{G}, 100 \mathrm{~mm})$ connected to nerve stimulator $(2 \mathrm{~Hz}, 1.5 \mathrm{~mA}$ ) was inserted usually 4.5 to 5 $\mathrm{cm}$ lateral to midline (to L3) and inclined a little cranially to pass between the L4 and L5 transverse processes advancing a further 1.5 to $2.0 \mathrm{~cm}$ past the transverse process. Once the needle accurately placed, permit the motor response with a current below $0.5 \mathrm{~mA}$ and negative blood aspiration, the LA injected gradually and distributed around the lumbar plexus with frequent aspiration for blood each $5 \mathrm{ml}$ (fig 1). If within $9 \mathrm{~cm}$ from the skin, the stimulation of the lumbar plexus does not take place, the needle was removed from the skin and reinserted after increasing angulations of the needle by $10^{0}$ laterally.

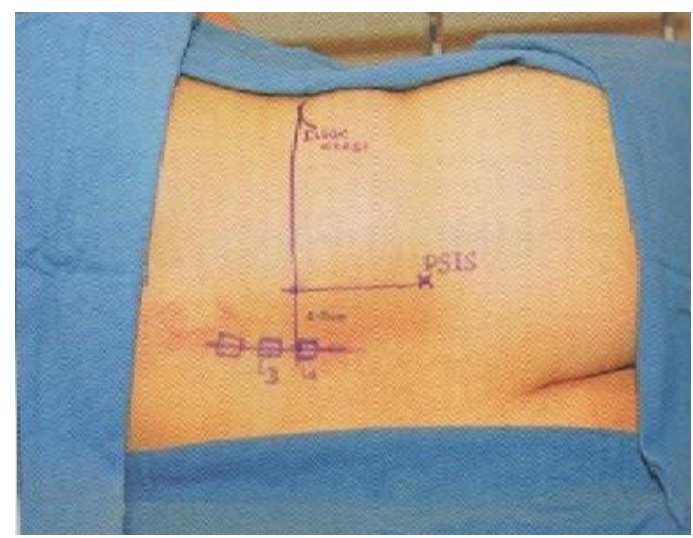

Fig. 1: Posterior approach towards lumbar plexus nerve block (psoas compartment block)

The sciatic nerve block was performed by classical Labat's approach to accomplish complete anaesthesia of the lower limb followed by psoas compartment block. The patients with same lateral decubitus position (Sim's position) two lines were drawn: the first from the greater trochanter to the sacral hiatus and second from greater trochanter to the posterosuperior iliac spine (PSIS). A perpendicular was drawn from the midpoint of the greater trochanter-PSIS line, caudad that intersected the greater trochanter-sacral hiatus line, which represent the insertion point. Under all safety measures the insulated needle was connected to nerve stimulator $(1.5 \mathrm{~mA}, 2 \mathrm{~Hz})$ which was at right angles to the skin. The needle was injected after encountering gluteal muscle stimulation to elicit a motor response in the 
distal ankle or the foot. The stimulation was reduced to $<0.5 \mathrm{~mA}$. The LA was injected slowly with frequent aspiration for blood each $5 \mathrm{ml}$ after negative blood aspiration (fig 2).

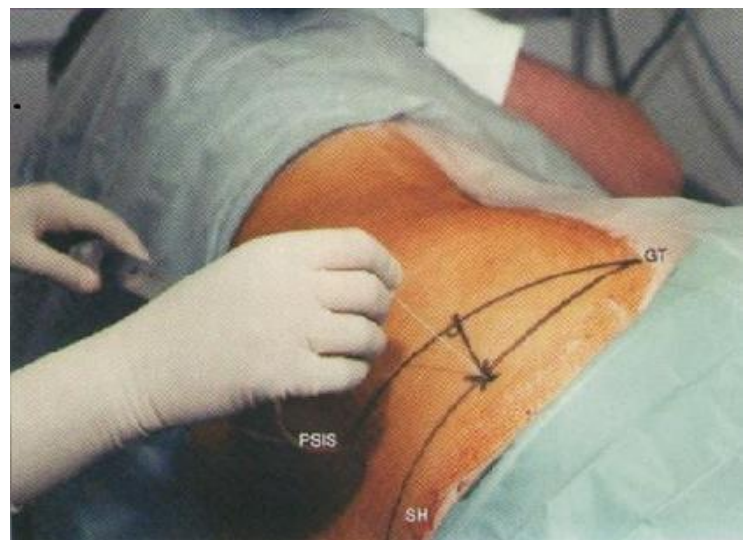

\section{Fig. 2: Sciatic Nerve Block By Classical Labat's approach}

In group B, spinal anaesthesia was carried out in the L3-L4 interspaced with the patient in lateral decubitus position with knee flexed where shoulder and hip lies in same plane. Lumbar puncture was done in anterior superior iliac spine, midline at L3 L4 space with $25 \mathrm{G}$ spinal needle. After confirming clear, free, continuous flow of cerebrospinal fluid, injection bupivacaine $(0.5 \%)$ (Heavy) $3 \mathrm{cc}$ was given. Patient was made supine immediately. In all sixty patients we inserted epidural catheter for post-operative analgesia and as an adjuvant for prolonged duration of surgery.

In both the groups, by using pinprick tests, the sensory block level was tested and graded as: Excellentno pain sensations; Good- mild pain sensations present but tolerable; Fair-sensations present required sedatives and analgesics or epidural supplementation and Poorpain sensations present and intolerable. Motor block level was evaluated with the Modified Bromage scale (Grade 0-Able to lift leg against gravity, grade 1-Able to flex knees but unable to lift legs, grade 2-Able to move feet but unable to flex knees, grade 3-No movement at all). Obturator muscle strength was assessed with the patient supine by determining hip adductor strength using above scale. At the time of injecting the drug, the onset of sensory and motor block was measured. The requirement of epidural supplementation, efficacy and duration of sensory and motor block were recorded. The pulse rate and blood pressure were measured before and after premedication, as well as before and after block at 5 min and then at intervals of 10 minutes, till the end of $180 \mathrm{~min}$ or at the end of surgery. Any complications observed in both groups were noted. Also duration of postoperative analgesia was recorded when the first rescue analgesics were given postoperatively.

In present study, values were measured as mean \pm SD where appropriate. Students ' $t$ ' test and $Z$ test were applied for comparison between groups. Differences were considered to be statistically significant if $p$ value $<0.05$.

\section{Observations and Results}

Sixty patients were selected for the study, divided into group A and B. In group A there were $40 \%$ females and $60 \%$ males while in the group B there were $46.6 \%$ females $53.3 \%$ and males. The patient's mean age in group A and B were $47.34 \pm 13.83$ years and $46.22 \pm$ 14.18 years respectively. The most common surgery carried out in both the groups was tibia nailing, distal femoral plating and proximal femoral nail. Other type of surgery performed in the study was trochanteric femur nailing, patella TBW and tibia plating. The group $\mathrm{A}$ and $\mathrm{B}$ were comparable with regard to demographic data (age, sex, physical status) and type of surgeries and difference was not statistically significant.

The onset of sensory and motor block was earlier in group B (SA) compared to group A (LPB + Sciatic Nerve Block), which was statistically significant. The mean duration was significantly prolonged in group A than in group B. The efficacy of sensory blockade was comparable in both the groups and was statistically significant. The complete motor blockade was observed in $90 \%$ of group A patients with bromage scales 1, 2 and 3 while $97 \%$ of group B patients. None of the cases required epidural supplementation in both the groups. The results of characteristics of sensory and motor blockade were depicted in Table 1. 
Table 1: Characteristics of sensory and motor blockade

\begin{tabular}{|c|c|c|c|}
\hline Characteristics & Group A & Group B & p-value \\
\hline Onset of sensory block & $5.4 \pm 1.28$ & $3.03 \pm 0.69$ & \multirow{4}{*}{$\begin{array}{c}<0.0001 \\
\mathrm{~S}\end{array}$} \\
\hline Onset of motor block & $15.3 \pm 2.32$ & $7.393 \pm 0.34$ & \\
\hline Duration of sensory block & $8.1 \pm 0.96$ & $2.3 \pm 0.58$ & \\
\hline Duration of motor block & $5.45 \pm 1.0$ & $2.81 \pm 0.77$ & \\
\hline \multicolumn{4}{|c|}{ Efficacy of sensory blockade } \\
\hline Fair & 6 & 5 & \multirow{3}{*}{$\begin{array}{c}\mathrm{P}>0.05 \\
\mathrm{NS}\end{array}$} \\
\hline Good & 18 & 20 & \\
\hline Excellent & 6 & 5 & \\
\hline \multicolumn{4}{|c|}{ Efficacy of motor block (Modified Bromage Scale) } \\
\hline 0 & 0 & 0 & \multirow{4}{*}{$\begin{array}{c}\mathrm{P}>0.05 \\
\mathrm{NS}\end{array}$} \\
\hline 1 & 0 & 0 & \\
\hline 2 & 3 & 1 & \\
\hline 3 & 27 & 29 & \\
\hline
\end{tabular}

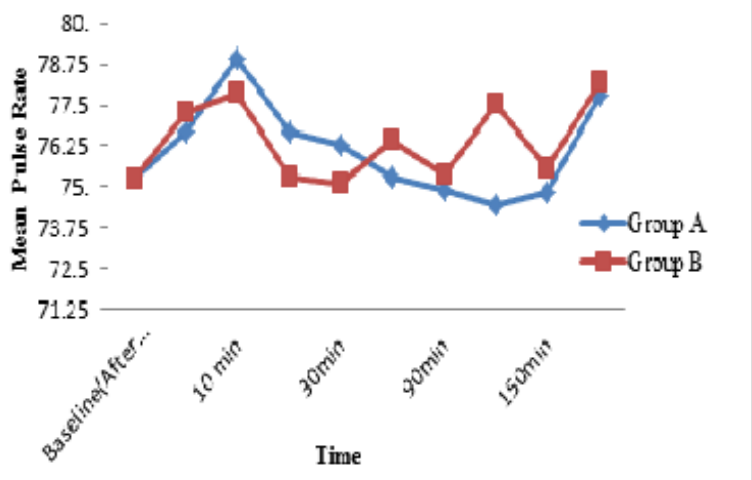

\section{Fig. 1: Variation in mean pulse rate at various periods}

Fig. 1 represents the mean pulse rate of all the patients' changes at all time intervals was found to be statistically insignificant

We found significant changes in mean systolic, diastolic and mean arterial blood pressure at various periods as compared with the baseline values in group B patients (Fig 2). There was significant fall in blood pressure after induction as compared to its baseline values in group B patients. These indicate that incidence of hypotension was more with group B than group A and which was statistically significant.

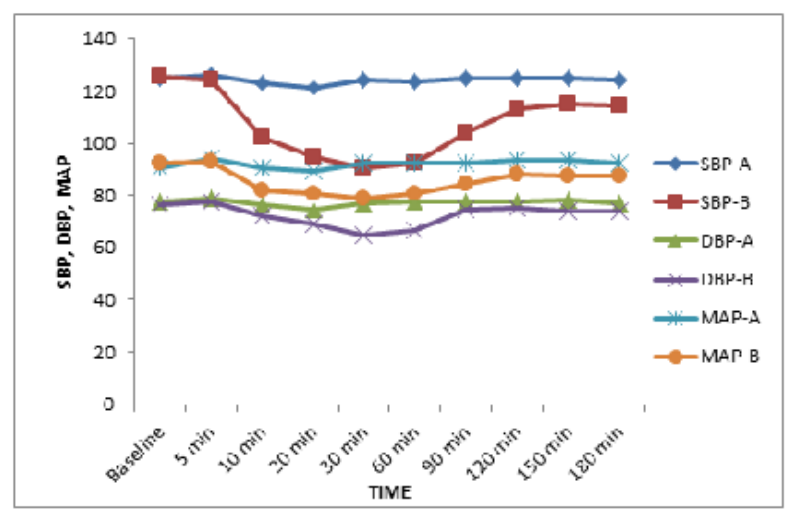

Fig 2: Changes in SBP, DBP and MAP at various periods

Group A had longer duration of post-operative analgesia (7.76 $\pm 0.86 \mathrm{hrs})$ compared to group B $(4.03 \pm 0.39 \mathrm{hrs})$. In both groups post-operative analgesia was further extended by epidural top ups for $48 \mathrm{Hrs}$. In present study, no major complications were observed in both the groups. 


\section{Discussion}

For lower limb surgery, spinal anaesthesia, epidural anaesthesia and general anaesthesia are given routinely. However in case of very high risk patients, these methods are associated with increased rate of complications like hypotension. Lumbar plexus block can be used in whom avoidance of bilateral lower extremity block or sympathectomy accompanying neuraxial block is contraindicated. As per the studies of De Visme et al, ${ }^{7}$ Buckenmaier et $\mathrm{al}^{8}$ and Gaillat et al, ${ }^{9}$ Lumbar plexus block was combined with sacral plexus block is the only agent of anaesthesia for lower extremity surgeries. Hence it is believed that combining psoas compartment block and sciatic nerve block can help to perform surgeries on the entire lower limb as they block the lumbar as well as the sacral components. In addition, it allows tourniquet application that is commonly employed in majority of lower limb orthopedic surgeries.

The present research reveals the usefulness of combined psoas compartment and sciatic nerve block for adult patients for lower limb surgeries. It can be performed using Labat's approach with the help of nerve locator. It has been suggested that posterior approach for blocking psoas compartment impede the three main components of lumbar plexus. ${ }^{10-13}$ So this approach was found to be a good option in our patients, as it is more efficient in blocking the obturator nerve that supplies the anteromedial capsule of the lower extremities. The combination of these two approaches from the posterior aspect facilitate to conduct lower limb surgeries as both components were blocked without changing the position of the patient.

In our study, demographic data of the patients (age, sex and ASA grade) being comparable has no influence on outcome of the study. Among the sixty cases studied here, the number of female patients was less because most of the cases studied were traumatic and the prevalence of RTA was more in youth and in males than females. ${ }^{14,15}$ The onset of sensory block was evaluated from the time of injecting the drug up to loss of complete sensations. It was assessed using pinprick method and onset of analgesia was assessed after complete loss of pinprick sensations. The group B had early onset of sensory block than group $\mathrm{A}$ and the statistically significant difference was observed. Hence it was inferred that onset of analgesia was faster in spinal anesthesia compared to LPB + sciatic nerve block group. This result can be explained by the fact that local anesthetic solution must cross different anatomical barriers such as fibrous tissue and nerve sheaths before reaching the site of action in peripheral nerves. In spinal anaesthesia as there is direct contact with the nerve by the drug in subarachnoid space, the onset is quick. Thus in LPB + sciatic nerve block, drug diffuses in the larger space surrounding the plexus requiring longer time for onset of action. Motor blockade was evaluated with reference to the modified
Bromage Scale. ${ }^{16}$ Bromage scale of 2 or decrease in muscle power in the anaesthetized limb shows onset of motor blockade. Onset of motor block was quicker in group B compared to group A and which was statistically significant.

The duration of sensory and motor block was longer in group A than in group B, the difference was statistically significant as like the studies of Shrivastava et al. ${ }^{17}$ Hevia-Sanchez et al. ${ }^{18}$ Our study also supports prolonged duration of analgesia in $\mathrm{PCB}+\mathrm{SCB}$ group. The efficiency of sensory and motor blockade the capability of anesthesia was inferred and comparable among the groups. In the study of De Visme et $\mathrm{al}^{7}$ and Türker et $\mathrm{al}^{19}$ patients observed with inadequate anaesthesia and required general anaesthetic agents, sedatives or opioids. In our study, by giving sacral nerve block in addition to psoas compartment block, we overcame insufficient anaesthesia and avoided respiratory depressant drugs and general anaesthesia.

There was no statistically significant difference observed in pulse rate when comparing two groups. The results of the current studies support the data of Atim and et al. ${ }^{20}$ In previous reports, decreased heart rate was observed in patients who underwent psoas compartment block. This change was explained by a possible central neuraxial spread of the psoas compartment block because of large volumes injection of local anesthetic agents. ${ }^{21}$ We observed statistically significant fall in BP in group B as compared to their baseline values, while there was no change in group A in terms of systolic and diastolic blood pressure. So there was a significant fall in mean arterial pressure in group B patients as compared to their baseline values. Our study groups received significantly lower doses of bupivacaine $(0.25 \%$ $50 \mathrm{cc})$ unlike Leeuw et $\mathrm{al}^{22}$ study. So less hemodynamic alterations were observed in our LPB + sciatic nerve block group and there was significant hypotension observed in SA group. Our result correlates with different studies done by different authors. ${ }^{7,23,24}$ In our study bradycardia and tachycardia was defined as pulse rate $<60 / \mathrm{min}$ or $<20 \%$ and $>100 / \mathrm{min}$ or $>20 \%$ from the baseline respectively. If systolic BP decreased by $30 \%$ or diastolic decreased by $15 \%$ from the baseline results in hypotension and it was treated with adequate IV fluids and injection mephentaramine $3 \mathrm{mg}$.

The need of postoperative opiod requirement was much less in group A than B because the duration of postoperative analgesia was longer in group A, and found to be statistically significant. Besides, the time required for the first postoperative epidural supplementation in group A was delayed compared to group B. The results of our study were similar as observed by Moreno and Casalia et al..$^{23}$

In present study, we did not find any significant complications and hemodynamic instability during the surgery and postoperatively. Serious complications of sciatic nerve block were rare. Though there were theoretical concerns of injury to vascular structures and 
muscles. Residual dysthesias for periods of 1 to 3 days were not uncommon but usually resolve. There was no postoperative complication seen in group A patients, whereas in 2 patients of group B urinary retention was seen postoperatively as well as 4 patients complained of postoperative nausea and vomiting. Our results were same as found by Hinman $\mathrm{F}^{25}$ in their study.

\section{Conclusion}

Based on the results obtained from the study, it has been concluded that for surgeries of lower extremities of high risk and elderly patients, the combined psoas compartment block and sciatic nerve block is a useful choice to existing anaesthesia techniques.

Also the present study suggested that combined psoas compartment block and sciatic nerve block is a novel technique which provided effective intraoperative anaesthesia and postoperative analgesia. It takes care of sparing sacral plexus distribution area and patients remain hemodynamically stable throughout the procedure with longer duration of analgesia making them more comfortable.

\section{Acknowledgement}

We sincerely thank the department of anaesthesiology, surgery, other staff of operation theatre and administration of Dr. V.M. Government Medical College, Solapur, Maharashtra, for permission to study and providing necessary facilities to carry out the work.

\section{References}

1. http://data.worldbank.org/indicator/SP.POP.65UP.TO.ZS

2. Fowler SJ, Symons J, Sabato S and Myles PS. Epidural analgesia compared with peripheral nerve blockade after major knee surgery: a systematic review and meta-analysis of randomized trials. British Journal of Anaesthesia 2008;100 (2):154-64.

3. Indelli PF, Grant SA, Nielsen K, Vail TP: Regional anesthesia in hip surgery. Clin Orthop Relat Res 2005;441:250-5.

4. Imbelloni LE, Gouveia MA, Cordeiro JA: Continuous spinal anesthesia versus combined spinal epidural block for major orthopedic surgery: prospective randomized study. Sao Paulo Med J 2009;127:7-11.

5. Peyton PJ: Complications of continuous spinal anesthesia. Anaesth Intensive Care 1992;20:417-25.

6. Montes FR, Zarate E, Grueso R, Giraldo JC, et al.: Comparison of spinal anesthesia with combined sciaticfemoral nerve block for outpatient knee arthroscopy. J Clin Anesth. 2008; 20:415-20.

7. De Visme V, Picart F, Le JouanR, Legrand A, Savry C, Morin V Combined lumbar and sacral plexus block compared with plain bupivacaine spinal anesthesia for hip fractures in the elderly. RegAnesth Pain Med 2000; 25:15862.

8. Buckenmaier CC, 3rd, Xenos JS, Nilsen SM. Lumbar plexus block with perineural catheter and sciatic nerve block for total hip arthroplasty. J Arthroplasty 2002;17:499-502.

9. Gaillat F, Thibault S, Scemama F, Joly F, Paladini M, Auffray J. Bloc lombaire et sciatique posterieur pour fracture du col du femur du sujet age: experience clinique. Cah Anesthesiol 2002;50:99-104.
10. Morgan GE, Mikhail MS, Murray MJ. Peripheral nerve blocks. In: Morgan GE, Mikhail MS, Murray MJ, editors. Clinical Anesthesiology 4th ed. Singapore: McGraw-Hill Companies; 2008;324-58.

11. Wedel DJ, Horlocker TT. Nerve blocks. In: Miller RD, Eriksson LI, Fleischer LA, Wiener-Kronish JP, Young WL, editors. Miller's Anesthesia. Vol. 1 and 2. 7th ed. New York: Churchill Livingstone; 2010;1639-72.

12. Macalou D, Trueck S, Meuret P, Heck M, Vial F, Ouologuem S, et al. Postoperative analgesia after total knee replacement: The effect of an obturator nerve block added to the femoral 3-in-1 nerve block. Anesth Analg 2004;99:251-4.

13. McNamee DA, Parks L, Milligan KR. Post-operative analgesia following total knee replacement: An evaluation of the addition of an obturator nerve block to combine femoral and sciatic nerve block. Acta Anaesthesiol Scand 2002;46:95-9.

14. Park's textbook of preventive and social medicine $-21^{\text {th }}$ edition 2011-pg 375.

15. Youth and Road accidents- Role of health education - Dr. H. C. Agarwal $19^{\text {th }}$ Jan 1985- Swasth Hind.

16. Pramila Bajaj. Clinical anaesthesia- $2^{\text {nd }}$ edition, 2001.

17. Srivastava U, Kumar A, Saxena S, Naz A, Goyal V, Mehrotra R. Lumbar plexus block for postoperative analgesia following hip surgery, a comparison of " 3 -in-1" and psoas compartment block. Indian J Anaesth. 2007;12730.

18. Hevia-Sanchez V, Bermejo-Alverez MA, Hevia-Mendoz A, Fervienza F, Franch M, Diaz ML. Posterior block of lumbar plexus for postoperative analgesia after hip arthroplasty. Rev Esp Anestesiol REanim. 2002;49:507-11.

19. Turker G, Uckunkaya N, Yavascaoglu B, Yilmazlar A, Ozcelik S. Comparison of the catheter-technique psoas compartment block and the epidural block for analgesia in partial hip replacement surgery. Acta Anaesthesiol Scand 2003;47:30-6.

20. Atim A, Ergin A, Kurt E, Ozdemiroglu Y, and Guzeldemir E: Comparisin of sciatic psoas compartment block and sciatic femoral 3-in-1 block for knee arthroscopy. J Clin Anesth 2007;19:591-5.

21. Ganidagli S, Cendiz M, Baysal Z, Baktiroglu L, Sarban S. The comparison of two lower extremity block techniques combined with sciatic block: 3-in-1 femoral block vs. psoas compartment block. Int J Clin Pract 2005;59:771-6.

22. De Leeuw MA, Slagt C, Hoeksema M, Zuurmond WW, Perez RS. Hemodynamic Changes during a Combined Psoas Compartment-Sciatic Nerve Block for Elective Orthopedic Surgery Anesth Analg. 2011 Mar;112(3):719-24.

23. Moreno M, and Casalia AG: Techniques in regional anesthesia and pain management, Vol.10, Issue 4, Peripheral Nerve Blocks, Lumbar Plexus Anesthesia: Psoas compartment Block. Elsevier Inc. 2006;145-9.

24. Casati A, Fanelli G, Beccaric P, Aldegheri G, Berti M, Senatore R, Torri G: Cardiovascular changes for two different anaesthetic techniques for unilateral leg surgery. Acta Anaesthesiol Scand 1998;42:80-4.

25. Hinman F. Postoperative over distension of the bladder. Surg Gynecol Obstet1976;142:901-2. 